\title{
RESTRICTED NONCOMMUTATIVE JORDAN ALGEBRAS OF CHARACTERISTIC $p$
}

\author{
R. D. SCHAFER ${ }^{1}$
}

Noncommutative Jordan algebras over a field $F$ are defined by the identities

$$
\begin{aligned}
(x a) x & =x(a x), \\
\left(x^{2} a\right) x & =x^{2}(a x) .
\end{aligned}
$$

In $[7]^{2}$ we obtained a satisfactory structure theory for these algebras of finite dimension over $F$ of characteristic 0 by proving that they are trace-admissible. Recent examples by L. A. Kokoris [4] show that the algebras satisfying (1) and (2) are not in general traceadmissible if $F$ is of characteristic $p>2$.

It is natural to seek a generalization of (commutative) Jordan algebras of characteristic $p>2$ in which the algebras are trace-admissible. We find this generalization in the algebras satisfying (1), (2), and

$$
R_{x}^{p}=R_{x^{p}}
$$

where $R_{x}$ denotes the right multiplication determined by $x$. We shall call an algebra over $F$ of characteristic $p$, in which (1), (2), (3) are satisfied, a restricted noncommutative Jordan algebra (of characteristic $p$ ), using this terminology because of the analogy with restricted Lie algebras [2].

Commutative Jordan algebras of characteristic $p>2$ satisfy (3). For in any special Jordan algebra, the right multiplication is $R_{x}^{+}$ $=1 / 2\left(R_{x}+L_{x}\right)$ where $R_{x}$ and $L_{x}$ are restrictions of right and left multiplications in an associative algebra. Then $\left(R_{x}^{+}\right)^{p}=1 / 2^{p}\left(R_{x}+L_{x}\right)^{p}$ $=1 / 2\left(R_{x}^{p}+L_{x}^{p}\right)$ since $\left[R_{x}, L_{x}\right]=0$. Hence $\left(R_{x}^{+}\right)^{p}=1 / 2\left(R_{x^{p}}+L_{x^{p}}\right)=R_{x^{p}}^{+}$ since powers in the special Jordan algebra and the associative algebra coincide. But $(3)$ is an identity, $(\cdots((a x) x) \cdots) x=a x^{p}$, in only two elements. Since the free (commutative) Jordan algebra of characteristic $\neq 2$ with two generators is special [9], identity (3) is satisfied in any (commutative) Jordan algebra of characteristic $p>2$. (Or one may use the formal identity

Presented to the Society August 28, 1957; received by the editors June 28, 1957.

1 This research was supported by a grant from the National Science Foundation.

2 Numbers in brackets refer to the references cited at the end of the paper. 


$$
R_{a^{n}}=1 / 2\left\{\left(R_{a}+\left(R_{a}^{2}-R_{a}^{2}\right)^{1 / 2}\right)^{n}+\left(R_{a}-\left(R_{a}^{2}-R_{a}^{2}\right)^{1 / 2}\right)^{n}\right\}
$$

in commutative Jordan algebras $\left[5\right.$, p. 256] and the fact that $\left[R_{a}, R_{a}{ }^{2}\right]$ $=0$ to see that $R_{a}^{p}=1 / 2\left\{\left(R_{a}+C\right)^{p}+\left(R_{a}-C\right)^{p}\right\}=1 / 2\left(R_{a}^{p}+C^{p}+R_{a}^{p}\right.$ $\left.-C^{p}\right)=R_{a}^{p}$.)

Alternative algebras (of arbitrary characteristic) satisfy (3), as well as (1) and (2). In fact, if $p=2$, then (1) and (3) imply the alternative law (so that (2) holds), and the theory of these algebras is well known. Our proofs are given for $p>2$, but the statements of the results include $p=2$ when applicable.

Lemma 1. Let $A$ be a restricted noncommutative Jordan algebra of characteristic p. If $x$ is nilpotent, then $R_{x}$ and $L_{x}$ are nilpotent.

For there is an integer $e$ such that $x^{p^{*}}=0$. Hence (3) implies that $R_{x}^{p^{p}}=0, R_{x}$ is nilpotent. Since $A^{+}$is a commutative Jordan algebra, we know that $\left(R_{x}^{+}\right)^{p^{f}}=0$ for some $f \geqq e$, or $1 / 2^{p^{f}}\left(R_{x}+L_{x}\right)^{p^{f}}$ $=1 / 2\left(R_{x}^{p f}+L_{x}^{p f}\right)=1 / 2 L_{x}^{p f}=0$ since $\left[R_{x}, L_{x}\right]=0$ by (1).

A noncommutative Jordan algebra $A$ is a nodal algebra [8] in case $A=F 1+N$ where $N$ is a subspace consisting of the nilpotent elements of $A$, and $x \cdot y=1 / 2(x y+y x) \in N$ for all $x, y \in N$, but there exist $x, y \in N$ such that $x y \notin N$. In [4] Kokoris constructs simple nodal noncommutative Jordan algebras for each characteristic $p>2$. We use here the notation of $[8, \S 3]$. In particular, we write the descending chain of subspaces

$$
N_{1} \supset N_{2} \supset N_{3} \supset \cdots \supset N_{k} \supset N_{k+1}=0
$$

as in $[8,(3)]$.

Leмma 2. Let $A=F 1+N$ be a noncommutative Jordan algebra of characteristic $\neq 2$. If $z \in N, w \in N_{2}$, and $R_{w}$ is nilpotent, then $R_{z}^{+}+R_{\text {o }}$ is nilpotent.

We prove by induction on $h$ that, for any $i$,

$$
N_{i}\left(R_{z}^{+}+R_{w}\right)^{h} \subseteq N_{i+1}+N_{i} R_{w}^{h} .
$$

The case $h=0$ is clear. Assuming (6), we have $N_{i}\left(R_{z}^{+}+R_{w}\right)^{h+1}$ $\subseteq\left(N_{i+1}+N_{i} R_{w}^{h}\right)\left(R_{z}^{+}+R_{w}\right) \subseteq N_{i+2}+\left(N_{i} R_{w}^{h}\right) \cdot N+N_{i+1} N_{2}+N_{i} R_{w}^{h+1}$. But $N_{i} R_{w}^{h} \subseteq N_{i}$ and $N_{i+1} N_{2} \subseteq N_{i+1}$ by [8, Theorem 5(b)]. Hence $N_{i}\left(R_{z}^{+}+R_{w}\right)^{h+1} \subseteq N_{i+1}+N_{i} R_{w}^{h+1}$. Let $R_{w}^{t}=0$. Then (6) implies

$$
N_{i}\left(R_{z}^{+}+R_{w}\right)^{t} \subseteq N_{i+1}^{\top}
$$

for any $i$. Hence

$$
N_{1}\left(R_{z}^{+}+R_{w}\right)^{k t} \subseteq N_{2}\left(R_{z}^{+}+R_{w}\right)^{(k-1) t} \subseteq \cdots \subseteq N_{k}\left(R_{z}^{+}+R_{w}\right)^{t} \subseteq N_{k+1}=0
$$


by (5), and $A\left(R_{z}^{+}+R_{w}\right)^{k t+1}=\left(F 1+N_{1}\right)\left(R_{z}^{+}+R_{w}\right)^{k t+1} \subseteq N_{1}\left(R_{z}^{+}+\right.$ $\left.R_{w}\right)^{k t}=0$.

Lemma 3. Let $A=F 1+N$ be a noncommutative Jordan algebra of characteristic $\neq 2$. If $x, y \in N$ are such that $R_{x \cdot y}$ is nilpotent, then $x y \in N$.

Let $y^{r} \neq 0, y^{r+1}=0$. Then $x y=\lambda 1+z, \lambda \in F, z \in N$, and $y x=-\lambda 1$ $+(2 x \cdot y-z)$. Flexibility implies $\left(x, y, y^{r}\right)+\left(y^{r}, y, x\right)=0$, so that $\lambda y^{r}+z y^{r}-x y^{r+1}+y^{r+1} x+\lambda y^{r}-2 y^{r}(x \cdot y)+y^{r} z=0$, or $y^{r}\left(\lambda I+R_{z}^{+}-R_{x \cdot y}\right)$ $=0$. Hence $\lambda I+R_{z}^{+}-R_{x \cdot y}$ is singular. Let $w=-x \cdot y$ in Lemma 2 , so that $R_{z}^{+}-R_{x \cdot y}$ is nilpotent. This forces $\lambda=0, x y \in N$.

Lemmas 1 and 3 imply

Theorem 1. A restricted noncommutative Jordan algebra of characteristic $p$ cannot be a nodal algebra.

If $A$ is a restricted noncommutative Jordan algebra of characteristic $p$, then so is $A_{K}$ where $K$ is any extension of $F$. For there are at least $p$ distinct elements in $F$, sufficiently many to effect the linearization of (3). Using the linearized form of (3), together with the intermediate identities obtained in the linearization, one can verify (3) in $A_{K}$. Then [8, Theorems 2, 3] imply

TheORem 2. Any restricted noncommutative Jordan algebra of characteristic $p$ is trace-admissible. If $p>2$, then the primitive trace function $\delta(x)$ of $A^{+}$is an admissible trace function for $A$, and the radical $N$ of $A$ consists of those elements $z$ satisfying $\delta(x z)=0$ for every $x$ in $A$.

R. H. Oehmke [6] has recently shown that any semisimple noncommutative Jordan algebra of characteristic $\neq 2,3$ is a direct sum of simple algebras, each of which is either a nodal algebra over its center or one of the simple algebras listed in [7]. Except for characteristic 3 his results are stronger than our Theorem 4 in [8]. In the present context we may apply [8, Theorem 4], however, to obtain

THEOREM 3. Any semisimple restricted noncommutative Jordan algebra $A$ of characteristic $p$ is uniquely expressible as a direct sum $A$ $=A_{1} \oplus \cdots \oplus A_{r}$ of simple ideals $A_{i}$. If $A$ is simple, then $A$ is one of the following:

(a) a simple (commutative) Jordan algebra of characteristic $p \neq 2$,

(b) a simple flexible algebra of degree two satisfying (3),

(c) an algebra $B(\lambda)$ where $B$ is a simple associative algebra over $F$ and $\lambda$ is an element $(\neq 1 / 2)$ in the prime field $P$ of $F$.

For not all of the simple quasiassociative algebras over $F$ need be restricted noncommutative Jordan algebras of characteristic $p$. Let 
$A$ be a simple quasiassociative algebra over $F$, and let $Z$ be the center of $A$. Then $A$ is a central simple quasiassociative algebra over $Z[1$, p. 586]. There exists an extension $K=Z(\lambda)$ of $Z$, and a central simple associative algebra $B$ over $K$, such that $A_{K} \cong B(\lambda)$. Let $R_{x}$ and $L_{x}$ denote right and left multiplications in $B$ so that the right multiplication $R_{x}^{*}$ in $B(\lambda) \cong A_{K}$ is given by $R_{x}^{*}=\lambda R_{x}+(1-\lambda) L_{x}$. Then $\left(R_{x}^{*}\right)^{p}=R_{x^{p}}^{*}$ implies $\lambda^{p} R_{x}^{p}+\left(1-\lambda^{p}\right) L_{x}^{p}=\lambda R_{x^{p}}+(1-\lambda) L_{x}^{p}$, or $\left(\lambda^{p}-\lambda\right)\left(R_{x}^{p}-L_{x}^{p}\right)=0$, so that either

$$
\lambda^{p}-\lambda=0,
$$

or $R_{x}^{p}=L_{x^{p}}$ for every $x$ in $B$. In the latter case, $x^{p}$ is in the center $K$ of the central simple associative algebra $B$ for every $x$ in $B$. But then it is well-known (for example, see [3, p. 219]) that $B$ is commutative. In this case $A$ is trivially included in the classification of simple algebras. Hence we may assume that (8) holds, so that $\lambda$ is an element of the prime field $P$ of characteristic $p$. Hence $K=Z(\lambda)=Z$, and $B$ is a simple associative algebra over $F, A=B(\lambda)$. Conversely any such algebra satisfies (3).

A classification of the algebras (b) seems to be as complicated a matter as that of the classification of simple flexible algebras of degree two without the restriction (3). If $Q$ is a quaternion algebra, then $Q(\lambda)$ satisfies (3) if and only if $\lambda$ is in the prime field $P$ of characteristic $p$, as we have seen above. Since (3) is satisfied in any alternative algebra, the algebras (b) include all Cayley algebras $C$. But $C(\lambda)$ satisfies (3) if and only if $\lambda \in P$ (for the "only if" part of this statement we consider the subalgebra $Q(\lambda)$ above).

\section{REFERENCES}

1. A. A. Albert, Power-associative rings, Trans. Amer. Math. Soc. vol. 64 (1948) pp. 552-593.

2. N. Jacobson, Restricted Lie algebras of characteristic $p$, Trans. Amer. Math. Soc. vol. 50 (1941) pp. 15-25.

3. - Structure of rings, Amer. Math. Soc. Colloquium Publications, vol. 37, Providence, 1956.

4. L. A. Kokoris, Some nodal noncommutative Jordan algebras, Proc. Amer. Math. Soc. vol. 9 (1958) pp. 164-166.

5. W. H. Mills, $A$ theorem on the representation of Jordan algebras, Pacific J. Math. vol. 1 (1951) pp. 255-264.

6. R. H. Oehmke, On flexible rings, to appear in Ann. of Math.

7. R. D. Schafer, Noncommutative Jordan algebras of characteristic 0, Proc. Amer. Math. Soc. vol. 6 (1955) pp. 472-475.

8. - On noncommutative Jordan algebras, Proc. Amer. Math. Soc. vol. 9 (1958) pp. 110-117.

9. A. I. Shirshov, On special J-rings, Rec. Math. (Mat. Sbornik) N. S. voí. 38 (80) (1956) pp. 149-166.

The University of Connecticut 